\title{
Demographic Determinants Of Public Perceptions of Mental Illness In Heterogeneous Communities Of Lagos State Nigeria.
}

\author{
Akpunne, Bede C. \\ Department of Behavioral Studies \\ Redeemers University. Nigeria \\ Uzonwanne, Francis C. \\ Department of Behavioral Studies \\ Redeemers University. Nigeria
}

\begin{abstract}
Social responses to mental illness depend on factors such as the type of disorder, impact on social function, and cultural concepts and presumptions about the illness. Attitudes toward mental illness are different among families, ethnic groups, individuals, cultures, and countries and are often influenced by the cultural and religious teachings. There is a dearth of research on public perceptions of mental illness in heterogeneous Nigerian communities. A cross-sectional design was used. 210 adults randomly selected from six heterogeneous communities responded to perception of mental illness test (PerMIT) (acceptable reliability coefficient (Cronbach Alpha of .74). There was no observed significant difference in gender, age, educational background, and occupation. Significant difference was however observed in religion. The prevailing culture and misguided societal conclusions which stigmatize mental illness has overbearing influence on peoples' perception and attitude towards mental illness. Educational interventions and public enlightenment campaigns that debunk misconceptions about mental illness as well as encourage contact with affected persons could play a role in reducing stigma among Nigerians.
\end{abstract}

Keywords: Public perceptions, mental illness, heterogeneous communities, Nigeria.

\section{INTRODUCTION}

While mental health care underwent this transition, public attitudes remained unchanged. Beliefs that had held sway for hundreds of years continued to stigmatize those who sought help for mental health problems, and doctors associated with the disease were also looked down upon. Social responses to mental illness depend on a variety of factors, including the type of disorder and its impact on social function, as well as cultural concepts and presuppositions about the illness. Concepts of mental illness are in turn influenced by values, religion, and the social context in which they are found ( $\mathrm{Ng}, 1997$, Adewuya et.al 2011, Dogra et.al. 2012,; Ikwuka et.al. 2014).

The World Health Organization (2010) regards mental health as a complicated phenomenon which relies in part on the successful implementation of public health efforts to control 
neuropsychiatric disorder such as depression, anxiety disorder, schizophrenia, and dementia, and is determined by environmental, psychological, biological and social factors. About 1500 million people global are estimated to be suffering from some kind of neuropsychiatric disorder, including behavioral, substance abuse and mental disorder. Thirty percent of them may be influenced by more than one neuropsychiatric sickness. $75 \%$ of those influenced live in creating nations like Nigeria WHO, (2010).

Mental illness often minimizes the usefulness of the individual to his or her family and loved ones. It also damages their self-image and force dependency upon relatives and society which may be difficult for them to accept, Mojoyinola, (2002).

\section{Public perceptions towards Mental Illness.}

Attitudes toward mental illness are different among families, ethnic groups, individuals, cultures, and countries and are influenced by the cultural and religious teachings. The way people interact with, help support and make available opportunities for individuals with mental illness is determined by their attitudes and beliefs toward mental illness. How people express their emotional difficulties and experience and psychological problems is affected by their attitudes and beliefs toward mental illness and whether they make known these signs and look for treatment (Mental Health Foundation, 2010).

Stigma often produces false information about people, fosters discriminatory acts against them and it is often used to convey prejudice and negative stereotypes, (Corrigan, Green, Lundin, Kubiak, \& Penn, 2001). People's personal knowledge concerning mental illness, being familiar with practices in the institution, past restrictions such as employment restrictions, adoption restrictions and health insurance restrictions, media stories, cultural labels, knowing and interacting with someone who is mentally ill will determine their attitudes and beliefs toward mental illness, (Corrigan et al., 2004; Wahl, 2003).

Self-stigma is common among individuals suffering from mental illness (Adewuya et.al 2011). A quarter of patients suffering from depression experience high self-stigma and the more the severity of the illness result from higher self-stigma, (Yen, Chen, Lee, Tang, Yen \& Ko, 2005). The negative labels that other people hold about people with mental illness are internalized by the mentally ill individuals (Corrigan, Watson \& Barr, 2006). Stigma encourages people to avoid, fear, discriminate and reject the mentally ill person as a result of their negative beliefs and attitudes, (Commission on Mental Health, 2003). Many people become ashamed and tend not to get treatment due to stigma and myths and poor perception about mental illness.

People believe that the mentally ill individuals are apt to be unintelligent and unfortunate, lack of willpower and personal weakness are believed to cause mental illness. The undesirable interpretation of mental illnesses in the media, whether in movies, newscasts or television have prolonged many mis-guided perceptions of mental illness, (CMHA, 2006). The mentally ill individuals are labeled due to their behavior, treatment, socioeconomic status, appearance and as a result of the adverse portrayal of mental illness in the media, (Scheffer, 2003).

Stigmatization leads to a compromised social stand and a mark of disgrace or humiliation. People perceive individuals living with mental illnesses as mad (Nguyen, 2013), crazy, stupid, useless, lazy, unsafe to be with, unintelligent, controlled by evil spirits, violent, always need to be supervised, volatile, not reliable, receivers of divine punishment, not responsible, without cognizant, incapable of getting married and raising children, incapable of working, increases in sickness throughout life and need to be hospitalized. Unfortunately, the widespread of these 
myths remain even among those that are supposed to provide treatment or health care service which the mentally ill individual needs (Robin \& Regier, 2001,; Canadian Mental Health Association, 2014). Perception of people on individuals with mental illness in heterogeneous Nigerian communities is scanty in literature, hence this study.

\section{Objectives}

- To examine the gender difference on public perceptionss of mental illness in heterogeneous communities of Lagos state.

- To ascertain the age difference on public perceptionss of mental illness in heterogeneous communities of Lagos state.

- To examine the educational background difference on public perceptionss of mental illness in heterogeneous communities of Lagos state.

- To ascertain the religion difference on public perceptionss of mental illness in heterogeneous communities of Lagos state.

- To find out the occupation difference on public perceptionss of mental illness in heterogeneous communities of Lagos state.

\section{Statement Of Hypotheses}

- There will be no significant gender difference on public perceptionss of mental illness in heterogeneous communities of Lagos state.

- There will be no significant age difference on public perceptionss of mental illness in heterogeneous communities of Lagos state.

- There will be no significant educational background difference on public perceptionss of mental illness in heterogeneous communities of Lagos state.

- There will be no significant religion difference on public perceptionss of mental illness in heterogeneous communities of Lagos state.

- There will be no occupation difference on public perceptionss of mental illness in heterogeneous communities of Lagos state.

\section{Justifications}

Findings of this study will be useful for public enlightenment about how to relate properly and management of people with mental illness in the society. It will also assist policy formulation on issues of stigmatization of individuals with mental illness in Nigeria.

\section{METHODOLOGY}

A cross sectional survey design was employed in the study. The population comprised of adult residents in six selected heterogeneous communities of Lagos State Nigeria. A purposive random sampling technique was adopted to select 210 out of 250 individual who participated in the study.

\section{Instrument}

A structured questionnaire tagged perception of mental illness test (PerMIT), with observed acceptable reliability coefficient (Cronbach Alpha of .74) was used for the study.

\section{Data Presentation and Analysis.}

The data was analyzed using SPSS package, such as simple percentage, t-test for independent groups and Analysis of Variance (ANOVA). 


\section{TEST OF HYPOTHESIS}

Hypothesis 1: There will be no significant gender difference on public perceptions of mental illness in heterogeneous communities of Lagos state.

Table 1: t-test of gender difference in perceptions of mental illness in Lagos state.

\begin{tabular}{|l|l|l|l|l|l|l|}
\hline Gender & N & Mean & S.D & df & t & $\boldsymbol{P}$ \\
\cline { 1 - 3 } Male & 85 & 37.68 & 5.21 & & & \\
\cline { 1 - 4 } Female & 125 & 37.83 & 5.50 & 208 & -0.20 & $>0.05$ \\
\hline
\end{tabular}

Table 1 shows that there is no significant gender difference (male $(t=(210) 37.68 p>0.05)$ and female $(t=(210) 37.83 \mathrm{p}>0.05)$ on public perceptions of mental illness in heterogeneous communities. This finding reveals that gender does not have a significant influence on people's perception about mental illness in heterogeneous communities.

Hypothesis 2: There will be no significant age difference on public perceptionss of mental illness in heterogeneous communities of Lagos state

Table 2: ANOVA of age difference in perceptions of mental illness in Lagos state.

\begin{tabular}{|c|c|c|c|c|c|c|c|}
\hline Age & $\mathbf{N}$ & Mean & S.D & df & $\mathbf{F}$ & $P$ & \\
\hline $18-24$ & 69 & 38.30 & 5.03 & \multirow{5}{*}{208} & \multirow{5}{*}{0.46} & \multirow{5}{*}{\multicolumn{2}{|c|}{$>0.05$}} \\
\hline $25-31$ & 51 & 37.55 & 5.22 & & & & \\
\hline 32-39 & 39 & 37.00 & 6.33 & & & & \\
\hline $40-47$ & 29 & 37.51 & 5.37 & & & & \\
\hline $48-55$ & 22 & 38.31 & 5.1 & & & & \\
\hline
\end{tabular}

Table 2 shows that there is no significant age difference on public perceptionss of mental illness in heterogeneous communities $(\mathrm{F}=(210) 0.46, \mathrm{P}>0.05)$. However, ages $48-55$ scored highest mean (38.31) followed by ages $18-24$ (mean = 38.30), ages $25-31$ (mean = 37.55), ages $40-47$ (mean $=37.51)$ and lastly ages $32-39$ (mean $=37.00)$. This result reveals a similar perception of mental illness among people of various ages. In other words people's chronological age does not significantly influence their perception about mental illness in heterogeneous communities.

3.1.3 Hypothesis 3: There will be no significant educational background difference on public perceptions of mental illness in heterogeneous communities of Lagos state.

Table 3: ANOVA of educational background difference in perceptions of mental illness in Lagos state.

\begin{tabular}{|c|c|c|c|c|c|c|}
\hline Educational Background & $\mathbf{N}$ & Mean & S.D & Df & $\mathbf{F}$ & $\mathbf{P}$ \\
\hline Primary education & 12 & 39.67 & 6.24 & \multirow{5}{*}{208} & \multirow{5}{*}{1.71} & \multirow{5}{*}{$>0.05$} \\
\hline Secondary education & 44 & 36.25 & 5.01 & & & \\
\hline B.Sc/B.A & 130 & 37.85 & 5.53 & & & \\
\hline Master/Ph.D & 17 & 40.00 & 4.24 & & & \\
\hline Others & 5 & 36.66 & 3.02 & & & \\
\hline
\end{tabular}


Table 3 shows that there is no significant educational background difference on public perceptionss of mental illness in heterogeneous communities $(F=(210), 1.71, P>0.05)$. However Master/Ph.D. scored highest mean score (40.00) followed by primary education level holders (mean $=39.67$ ), next is those with B.Sc. /B.A degree (mean = 37.85 and lastly secondary education (mean $=36.25)$. This result reveals a similar public perceptions of mental illness among people with different educational backgrounds. Hence people's educational background does not significantly influence their perception of mental illness in heterogeneous communities.

Hypothesis 4: There will be no significant religion difference on public perceptionss of mental illness in heterogeneous communities of Lagos state

Table 4: ANOVA of religion difference in perception of mental illness in Lagos state.

\begin{tabular}{|c|c|c|c|c|c|c|}
\hline Religion & $\mathbf{N}$ & Mean & S.D & Df & $\mathbf{F}$ & $P$ \\
\hline Christianity & 129 & 38.59 & 5.43 & \multirow{3}{*}{208} & \multirow{3}{*}{2.71} & \multirow{3}{*}{$<0.05$} \\
\hline Islam & 79 & 36.44 & 5.09 & & & \\
\hline Other religion & 5 & 36.00 & 5.35 & & & \\
\hline
\end{tabular}

Table 4 above shows that there is a significant religion difference on public perceptionss of mental illness in heterogeneous communities $(\mathrm{F}=(210) 2.71, \mathrm{P}<0.05)$. Christians manifested higher positive perception $($ mean $=38.59)$ than Muslims $($ mean $=36.44)$ and other religion (mean $=36.00)$. This result reveals that religion practiced have significant influence on public perceptions about mental illness in heterogeneous communities.

Hypothesis 5: There will be no significant occupational difference on public perceptionss of mental illness in heterogeneous communities of Lagos state.

Table 5: ANOVA of occupational difference on public perceptions mental illness.

\begin{tabular}{|c|c|c|c|c|c|c|}
\hline Occupation & $\mathbf{N}$ & Mean & S.D & Df & $\mathbf{F}$ & $\boldsymbol{P}$ \\
\hline Student & 64 & 38.45 & 5.48 & \multirow{4}{*}{208} & \multirow{4}{*}{2.18} & \multirow{4}{*}{$>0.05$} \\
\hline Informal employment & 62 & 36.32 & 5.54 & & & \\
\hline Formal employment & 79 & 38.34 & 5.05 & & & \\
\hline Unemployed & 5 & 38.00 & 4.41 & & & \\
\hline
\end{tabular}

Table 5 shows that there is no significant occupational difference on public perceptionss of mental illness in heterogeneous communities $(\mathrm{F}=(210) 2.18, \mathrm{P}>0.05)$. However, student scored higher $($ mean $=38.45)$, followed by those with formal employment (mean $=38.34)$, next is unemployed (mean $=38.00$ ) and lastly people with informal employment (mean $=36.32$ ). This result reveals that employment status does not significantly influence public perceptions about mental illness in heterogeneous communities.

\section{DISCUSSION}

The study revealed that there is no significant gender difference on public perceptionss of mental illness in heterogeneous communities. However in a related study, Bener \& Ghuloum (2011) reported that women held more to the cultural beliefs related to some aspects of mental illness than men, and that more women than men believed that mental illness is due to 
possession by evil spirits. Also, more women had belief that mental illness can be healed through traditional healing than men. Some of the women considered people with mental illness as dangerous; a belief also significantly lower in men. Men reported better attitude towards mental illness than women. Men were more willing to visit a psychiatrist for their emotional problems, while women preferred a traditional healer. Women were more afraid than men to talk to the mentally ill. Knowledge of common mental illnesses was generally poor, and it seemed to be lower among women. Men obtained more information than women from the media; women favored healthcare staff more than men did Bener \& Ghuloum (2011).

The study also revealed that there is no significant age difference on public perceptionss of mental illness in heterogeneous communities. In a study carried out by Dogra et.al. (2012) on the knowledge and attitude of Nigerian secondary school children on mental health and illness, it was found that Nigerian school children showed little knowledge and had negative attitude towards individuals with mental illness. They also kept social distance towards persons with mental health problems. Nigerian school children, as with Nigerian adults and young people in western countries, show stigma towards mental illness (Dogra et.al. 2012,; Ikwuka et.al. 2014) Children and adolescents widely report confronting problems with peer relationships due to the diagnosis of a mental illness. They also face isolation and ridicule from peers. Stereotypes that are associated with their diagnosis also do not go un noticed, many are bullied due to their mental health status (O'driscoll, Heary, Hennessy, \& Mckeague, 2012).

Furthermore, findings of this study show that there is no significant educational background difference on public perceptionss of mental illness in heterogeneous communities. In other words the level of education of the respondents had little or no influence on perception of the public about mental illness. This is however contrary to the findings of Ikwuka et.al (2014) who returned that there is a significant education background influence on people's attribution of mental illness to psychosocial causes. In other words Ikwuka et.al (2014) affirmed that respondents with higher education believed that the cause of mental illness was more of a psychosocial origin than a supernatural one. In a related study carried out on young adults, university and secondary school students as well as mental health professionals in Kenya, Mamah et.al (2013) affirmed that adolescents and young adults in Kenya were aware of mental illness in their communities, but had very limited knowledge of the meaning of specific psychiatric disorders and symptoms, and many of the respondents believed that the cause of mental illness was spiritual. Medical professionals have negative approach toward patients with mental illnesses and psychiatry, and they are found to have high percentage of myths (Canadian Mental Health Association, 2014). This study shows that when it comes to public view of mentally ill individual's vis-à-vis relating with them, the prevailing culture of the Nigerian society seems to have a stronger hold and realm of influence on the perception of people than their educational affiliations

This study also returned that there is a significant religion difference on public perceptions of mental illness heterogeneous communities. This is in support of the findings of Ikwuka et.al (2014) who examined causal attribution of mental illness in south-eastern Nigeria. It was returned that there is a significant attribution of supernatural cause of mental illness.

It also revealed that members of the Roman Catholic religious denomination returned a high psychosocial attribution to mental illness. In a study conducted on Christian churches in USA it was returned that religious teachings dismiss the existence of mental Illness attributing the cause to solely spiritual issues (Stanford, 2007). This by implication suggests a variance in the perception of people as a result of their religious affiliations. 
Finally this findings show that there is no significant occupation difference on public perceptionss of mental illness heterogeneous communities. Studies show that mentally ill individuals face lots of employment discriminations. According to Heather (2006) and (Clay 2012), people diagnosed of mental illness face a high level of employment discrimination. In some societies mental illness is considered as a greater barrier to employment than physical disabilities. Due to misconceptions associated to the phenomenon, mentally ill individuals are seen as violent, irrational, and incapable of doing things well and therefore people maintain a social distance from such individuals especially when it comes to employment.

Gureje, et.al. (2005) in a community study of knowledge of and attitude to mental illness in Nigeria returned that there is poor knowledge of causation of mental illness in Nigeria was common. That $96.5 \%$ of Nigerian had negative views of mental illness. There is a widespread believe that people with mental illness are dangerous because of their violent behavior. The stigmatization of mentally ill individuals is so widespread that most people in the society would not tolerate even basic social contacts with a mentally ill person. Gureje, et.al (2005) found that about $83 \%$ of community dwellers would be afraid to have a conversation with a mentally ill person and only $16.9 \%$ would consider marrying one. Socio-demographic predictors of both poor knowledge and intolerant attitude were generally very few. Negative attitudes to mental illness may be fuelled by notions of causation that suggest that affected people are in some way responsible for their illness, and by fear (Gureje, et.al 2005; Audu, et.al. 2011).

In a related study Audu, et.al (2011) examined stigmatization of people with mental illness among inhabitants of a rural community in northern Nigeria. It was found that despite the fact that mental illness is a common problem in society, people's perception of the mentally ill and community attitude towards them is still rather poor, making their rehabilitation and reintegration into society an uphill task.

Audu, et.al. (2011) returned a widespread ignorance about the cause, mode of transmission and remedies available for mental illness affirming that only $0.9 \%$ of respondents attributing mental illness to brain disease. The others attributed it to spiritual attack, punishment for evil doing and illicit psychoactive substance use, among other things. This shows a low level of awareness on what actually constitutes mental illness. A lot of cultural myths influence the perception of the average Nigerian on mental illness. This in turn manifest in the social disposition towards individual with mental illness.

Social stigma due to mental illness has over the years taken a global dimension. In 1999 the US surgeon general asserted that: "Powerful and pervasive, stigma prevents people from acknowledging their own mental health problems, much less disclosing them to others" (National Institute of Mental Health, 2009). Generally the public viewed people with mental illness as dangerous and less competent to handle their own affairs, with their harshest judgments reserved for people with schizophrenia and substance use disorders (USA General Social Survey 1996). Stigmatization of people with mental illness is highly prevalent among Nigerian children (Ronzoni, et.al 2010), and rampant in our community (Audu, et.al, 2011).

There is a need for adequate public education about the causes and mode of transmission of mental illness and the treatment options available in the community (Audu, et.al. 2011). Stigmatization has significant influence on the public perceptions of mental illness in our society also it has increased the level of social distance observed by members of the general public about people with mental illness (Adewuya \& Makanjuola, 2008, Ronzoni, et.al, 2010, 
Audu, et.al, 2011, Vidojevic, et.al 2013). In the Chinese society, mentally ill individuals cannot legally marry (Spencer, 2003).

The result of this study revealed that there is no significant difference in demographic variables such as gender, age educational background, and occupation. In other words vast majority of members of the public have similar perception about mental illness. This can be anchored on the prevailing culture and value system of a society about the phenomena. The society has over the years attached stigma on being mentally ill. Some even view it as a consequence of a spiritual demeanor or an act of the gods or even a punishment for past mistakes inherited or committed by an individual. Hence culture has a lot to do with how a people view individuals with mental illness. However most culturally based view of the phenomenon is a reflection of misconceptions transferred from generations which has greatly influenced attitude of people towards individuals with mental illness.

There is scanty research on the perceptions of people living with mental illness (Gureje, et.al 2005). Hence people have because of lack of proper knowledge of this phenomenon held on to myths that have caused stigmatization fueled by misconceptions regarding the cause and acceptable social relationship with mentally ill people in the community. Another issue that has significant influence on public perceptions of mental illness is media coverage. Most coverage of mental illness is negative and pejorative depictions. Such negative depictions contribute to stigma and negative attitude in the public and mentally ill individuals. (Diefenbach, 1997,; Coverdale, Nairn, \& Claasen, 2002,; Edney, 2004)

Cultural meanings of illness have real consequences in terms of whether people are motivated to seek treatment, how they cope with their symptoms, how supportive their families and communities are, where they seek help (mental health specialist, primary care provider, clergy, and/or traditional healer), the pathways they take to get services, and how well they fare in treatment. The consequences can be grave - extreme distress, disability, and possibly, suicide when people with severe mental illness do not receive appropriate treatment.

Culture influences many aspects of mental illness, including how patients express and manifest their symptoms, their style of coping, their family and community supports, and their willingness to seek treatment. Likewise, the cultures of the clinician and the service system influence diagnosis, treatment, and service delivery. Cultural and social influences are not the only determinants of mental illness and patterns of service utilization for racial and ethnic minorities, but they do play important roles.

\section{CONCLUSIONS}

This research focused on demographic determinants of public perceptions of mental illness in heterogeneous communities of Lagos state Nigeria. The results returned revealed that:

- There is no significant gender difference on public perceptions of mental illness in heterogeneous communities.

- There is no significant age difference on public perceptions of mental illness in heterogeneous communities

$\circ$ There is a significant religion difference on public perceptions of mental illness in heterogeneous communities

- There is no significant educational background difference on public perceptions of mental illness heterogeneous communities 
- Finally there is no significant occupational difference on public perceptions of mental illness heterogeneous communities.

\section{RECOMMENDATIONS.}

Educational interventions and public enlightenment campaigns that debunk misconceptions about mental illness as well as encourage contact with affected persons could play a role in reducing stigma among Nigerians. There is need to enlighten the Nigerian media practitioners on how negative journalism promotes public misconceptions of mental illness. Media houses should use their instruments both print and electronic to paint correct pictures of mental illness devoid of prejudice, cultural myth, bias and writings which encourage social distance and discrimination against mental illness in order to eliminate the stigmatization.

\section{References}

[1] Adewuya, A.O., Makanjuola R.O.A.,(2008). Social distance towards people with mental illness in Southwestern Nigeria. Australian New Zealand Journal of Psychiatry. Vol. 42 no. 5 389-395

[2] Adewuya, A.O., Erinfolami, A.O., Ola, B.A., (2011). Correlates of self-stigma among outpatients with mental illness in Lagos, Nigeria. Int. Journal of Social Psychiatry Vol. 57 no. 4 418-427

[3] Audu, I.A, Idris, S.H, Olisah, V.O, \& Sheikh, T.L, (2013).Stigmatization of people with mental illness among inhabitants of a rural community in northern Nigeria. International Journal of social psychiatry Vol. 59 no. 1 55-60

[4] Bener, A \& Ghuloum, S. (2011) Gender differences in the knowledge, attitude and practice towards mental health illness in a rapidly developing Arab society. Intentional Journal of Social Psychiatry Vol. 57 no. 5 480-486

[5] Canadian Mental Health Association (2006). Understanding mental illness. Available online at:"http://www.cmha.ca/bins/content_page.asp?cid=3\&lang=1"lang=1

[6] Clay, L (2012),’Stigma hurts job prospects". Sydney Morning Herald.

[7] Corrigan, P. W. (2004), "Target-Specific Stigma Change: A Strategy for Impacting Mental IllnessStigma," Psychiatric rehabilitation journal, Vol. 28, No. 2, pp. 113-121.

[8] Corrigan, P.W., Watson, A.C., \& Barr, L. (2006). The Self-stigma of Mental Illness: Implications for Self-esteem and Self-efficacy. Journal of Social and Clinical Psychology, 25, 875-884.

[9] Corrigan, P. W., Green, A., Lundin, R., Kubiak, M. A., \& Penn, D. L. (2001). Familiarity withand social distance from people who have serious mental illness. Psychiatric Services, 52, 953-958.

[10] Coverdale, J.; Nairn, R; Claasen, D, (2002). "Depictions of mental illness in print media: A prospective national sample". Australian and New Zealand Journal of Psychiatry 36 (5): 697-700.

[11] Diefenbach, D.L. (1997). "The portrayal of mental illness on prime-time television". Journal of Community Psychology 25 (3): 289-302.

[12] Dogra N., Omigbodun, O., Adedokun, T. , Bella, T, Ronzoni, P., \& Adesokan, A., (2012). Nigerian secondary school children's knowledge of and attitudes to mental health and illness. Clinical Child Psychology Psychiatry vol. 17 no. $3336-353$

[13] Edney, R.D. (2004). Mass Media and Mental Illness: a literature review. Canadian Mental Health Association

[14] Gureje O, Lasebikan V.O , Ephraim-Oluwanuga O. , \& Olley. B O., (2005). Community study of knowledge of and attitude to mental illness in Nigeria. The British Journal of Psychiatry 186: $436-441$ doi: 10.1192/bjp.186.5.436

[15] Heather S., (2006). "Mental illness and employment discrimination". Current Opinion in Psychiatry 19 (5): $522-6$.

[16] Ikwuka,U , Galbraith, N \& Nyatanga, L , (2014). Causal attribution of mental illness in south-eastern Nigeria, Int Journal of Social Psychiatry May 2014 vol. 60 no. 3 274-279

[17] Mamah, D, Striley, C.W., Ndetei, D.M., Mbwayo ,A.W., Mutiso.V.N., Khasakhala, L.I., \& Cottler, L.B., (2013). Knowledge of psychiatric terms and concepts among Kenyan youth: Analysis of focus group discussions. Transcultural Psychiatry, Vol. 50 no. 4 515-531 
Akpunne, B. C., \& Uzonwanne, F. C. (2015). Demographic Determinants of Public Perceptions of Mental Illness in Heteroneous Communities of Lagos State Nigeria. Advances in Social Sciences Research Journal, 2(1), 1-10.

[18] Mental Health Foundation, (2000) Mental Illness a global crisis, London.www.mentalhealth.org.uk/mental.illness

[19] National Institute of Mental Health, (2009). "A vision for the future". Mental health: A report for the Surgeon General. Pp. 451-8. ISBN978-0-16-050300-9

[20] Ng, C.H., (1997). The Stigma of Mental Illness in Asian Cultures. Australian and New Zealand Journal of Psychiatry, 31: p. 382-390.)

[21] Nguyen A. (2013) Cultural and Social Attitudes Towards Mental Illness in Ho Chi Minh City,

[22] O'driscoll, C., Heary, C., Hennessy, E., \& Mckeague, L. (2012). Explicit and implicit stigma towards peers with mental health problems in childhood and adolescence. Journal of Child Psychology \& Psychiatry, 53(10), 1054-1062.

[23] President's New Freedom Commission on Mental Health. (2003). Achieving the Promise:Transforming Mental Health Care in America. Rockville, MD: Author.

[24] Ronzoni, P., Dogra, N., Omigbodun, O., Bella,T., \& Atitola, O., (2010). Stigmatization of mental illness among nigerian schoolchildren, Int. Journal of Social Psychiatry Vol. 56 no. 5 507-514

[25] Scheffer, R. (2003). Addressing Stigma: Increasing public understanding of mental illness.Centre for Addiction and Mental Health.

[26] Spencer, R. (2003) “China relaxes laws on love and marriage” The Telegraph. Retrieved 24 October 2013

[27] Stanford, M.S. (2007). "Demon or disorder: A survey of attitudes toward mental illness in the Christian church". Mental Health, Religion \& Culture 10 (5): 445-9

[28] Vidojević I.M., Đurić Joči, D., \& Tošković, O., (2012). Comparative study of experienced and anticipated stigma in Serbia and the world Int. Journal of Social Psychiatry, Vol. 58 no. 4 355-361

[29] Wahl, O.F. (2003). News media portrayal of mental illness. American Behavioral Scientist, 46, 1594-1600.

[30] World Health Organisation, (2010); Mental Health and the Global Discourse; www.who.int

[31] Yen, C., Chen, C., Lee, Y., Tang ,T., Yen J., \& Ko C., (2005). Self-Stigma and Its Correlates among Outpatients with Depressive Disorders. Psychiatric Services, 56, 599-601. 